\title{
A single-center retrospective study of factors related to the effects of intravenous glucocorticoid therapy in moderate-to- severe and active thyroid-associated ophthalmopathy
}

\author{
Yang Wang ${ }^{1,2}$, Shuo Zhang ${ }^{1,2}$, Yidan Zhang ${ }^{1,2}$, Xingtong Liu ${ }^{1,2}$, Hao Gu ${ }^{3}$, Sisi Zhong ${ }^{1,2}$, Yazhuo Huang ${ }^{1,2}$,
} Sijie Fang ${ }^{1,2}$, Jing Sun ${ }^{1,2}$, Huifang Zhou ${ }^{1,2^{*}}$ and Xianqun Fan ${ }^{1,2^{*}}$

\begin{abstract}
Background: Intravenous glucocorticoids (ivGC) have been recommended as a first-line treatment of moderateto-severe and active thyroid-associated ophthalmopathy (TAO). However, not all patients are responsive to ivGC. The identification of potential factors used to predict their efficacy and the selection of suitable patients have both been lacking.

Methods: It was a single center retrospective study. Potential factors related to the effects of ivGC were analyzed using logistic regression in 90 consecutive patients with moderate-to-severe and active TAO, who received $4.5 \mathrm{~g}$ ivGC therapy. Response was defined as the achievement of at least three points of the overall response.
\end{abstract}

Results: Fifty-two (57.8\%) patients showed a positive response to ivGC therapy. Significant correlations were observed between the effects of ivGC and pretreatment clinical activity score (CAS), duration of eye symptoms, and restoration of euthyroidism. The two latter factors were both independent. The duration of eye symptoms was negatively correlated with the effects of ivGC, with an odds ratio (OR) of $0.984(p=0.012)$. Restoration of euthyroidism (OR $=3.282, p=0.039)$ and pretreatment CAS $(\mathrm{OR}=1.653, p<0.01)$ were both positively correlated with the effects of ivGC. The diagnostic accuracy of the duration of eye symptoms was $\leq 13$ months $(p=0.000)$, with a specificity of $76.9 \%$, and sensitivity of $65.8 \%$. The diagnostic accuracy of the pretreatment CAS was more than $2.5(p=0.000)$, with a specificity of $61.5 \%$ and sensitivity of $80.5 \%$. Besides, a multi-variables prediction model were established as well, which was better in the forecasting aspect with an area under curve of $0.784(p=0.000)$.

Conclusions: The duration of eye symptoms and restoration of euthyroidism are independent factors that are associated with the effects of ivGC. The following practical implications were inferred: firstly, the shorter the duration of eye symptoms, the more favorable the effects of ivGC therapy. Thus, prompt diagnosis and treatment (within 13 months) is important. Secondly, the restoration of euthyroidism improves the efficacy of ivGC. Thirdly, hope the multi-variables prediction model can be applied to clinical therapy in the future.

Keywords: Intravenous glucocorticoid therapy, Thyroid-associated ophthalmopathy, Duration of eye symptoms, Restoration of euthyroidism, Pretreatment clinical activity score

\footnotetext{
* Correspondence: fangzzfang@163.com; fanxq@sjtu.edu.cn

'Department of Ophthalmology, Ninth People's Hospital, Shanghai JiaoTong

University School of Medicine, No. 639 ZhiZaoJu Road, Shanghai 200011,

China

Full list of author information is available at the end of the article
} 


\section{Background}

Thyroid-associated ophthalmopathy (TAO) is an autoimmune inflammatory disorder that can lead to permanent vision loss. Common clinical manifestations include eyelid retraction (90\%), exophthalmos (60\%), and restricted extraocular motility (40\%) requiring medical intervention [1]. The various interventions can differ according to the characteristics of the patients and stages of the disease, and include the restoration of euthyroidism in all patients; selenium supplementation for those affected by mild TAO; immunosuppressive therapy and radiotherapy for active TAO; and decompression surgery for emergency or inactive TAO [2]. For active TAO, glucocorticoids continue to be the primary immunosuppressive therapy, and some steroidsparing regimens include rituximab and teprotumumab, whereas other regimens also prove to be effective [3]. Recent research has provided insight into potential differences in the effects of glucocorticoid therapy, based on various routes of administration (oral, intravenous, or local injection) [4-6]; doses; and schedules (weekly or daily) [7]. In the recent consensus statement of the European Group on Graves' Orbitopathy (EUGOGO), high-dose intravenous glucocorticoid (ivGC) therapy is recommended as the first-line treatment for moderate-to-severe and active TAO, and immediate therapy for sight-threatening TAO [2]. The response rate of ivGC appears to be approximately $70 \%-80 \%$ according to several randomized clinical trials $[7,8]$, suggesting that the remaining $20 \%-30 \%$ of all patients show no response and require additional therapy. Unfortunately, some of these patients have possibly missed their best treatment opportunities. Ineffective therapy can be a waste of resources. Thus, it is necessary to evaluate the prognoses and selection of suitable patients for ivGC. This study was designed to identify some of the potential factors associated with the effects of ivGC that can be used to predict their efficacy and facilitate the selection of suitable patients.

\section{Methods}

This retrospective study was approved by the Ethics Committee of Shanghai Ninth People's Hospital. It was in compliance with the tenets of the Declaration of Helsinki for clinical research.

\section{Study design and patients}

This study retrospectively reviewed a cohort of all consecutive patients diagnosed with active TAO, who had received $4.5 \mathrm{~g}$ intravenous methylprednisolone therapy. Patients were divided into two groups, based on their response to ivGC. The evaluation of response was based on the "overall response [5, 7]." Logistic regression models were then used to predict potential factors that were associated with the effects of ivGC therapy.
A total of 90 patients were included in the study, who presented to the Department of Ophthalmology, Shanghai Ninth People's Hospital, Affiliated Shanghai Jiao Tong University School of Medicine from December 2014 to December 2016. Diagnosis of TAO was based on the EUGOGO consensus [2]. Active TAO was defined by a.) clinical activity score (CAS) $\geq 3 / 7$; or b.) CAS $<3 / 7$, but with increased signal intensity of extraocular muscles on contrast-enhanced orbital magnetic resonance imaging (MRI). The CAS was also based on the EUGOGO consensus [2]. The protocol was as follows: $0.5 \mathrm{~g}$ per week for 6 weeks, followed by 0.25 g per week for 6 weeks. During the course of treatment, proton pump inhibitors or $\mathrm{H} 2$ receptor antagonists and calcium supplements were prescribed for every patient. Anti-thyroid drugs were used to restore and maintain euthyroidism. The detailed inclusion and exclusion criteria are presented in Table 1 and Table 2.

\section{Clinical assessment and response evaluation}

Demographic characteristics were compiled before treatment. The mean follow-up time was four weeks. Thyroid function and ophthalmic assessment that included the incidence of proptosis, eyelid width, diplopia, intraocular pressure, visual acuity, and CAS were compared before and after treatment ( 4 weeks after $4.5 \mathrm{~g}$ ivGC treatment). The classifications of diplopia were as follows: Grade 0, no diplopia; Grade 1, diplopia with horizontal or vertical gaze; Grade 2, intermittent diplopia in straight gaze; and Grade 3, constant diplopia in straight gaze. Eyelid width was recorded as the margin reflex distance (MRD), that is, the distance between the pupil center and eyelid margin. The MRD-1 was the distance between the pupil center and upper eyelid margin, whereas MRD-2 was the distance between the pupil center and lower eyelid margin.

Patients were divided into two groups, "responsive" and "unresponsive." The "responsive" group was defined as those manifesting at least three of the outcome measures (Table 3). Gender, age, duration of eye symptoms, smoking history, family history of TAO, history of impaired thyroid function, pretreatment CAS, and restoration of euthyroidism were analyzed to identify potential factors associated with the response to ivGC therapy. Gender was scored as male 1 and female 0 . Smoking history, family history of $\mathrm{TAO}$, and history of impaired thyroid function were scored

\section{Table 1 Inclusion criteria}

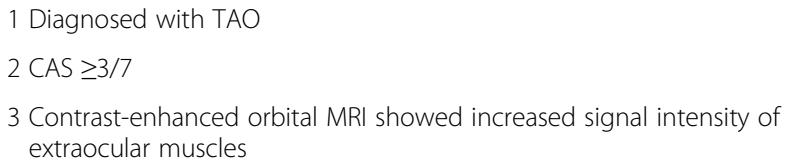

Patients should have met either criteria 2 or 3, together with criteria 1 and 4 to be included in the study. TAO thyroid-associated ophthalmopathy, CAS clinical activity score, $M R I$ magnetic resonance imaging 
Table 2 Exclusion criteria

1 Received orbital radiotherapy, decompression surgery, or other
immunosuppressive therapy within the previous 3 months or during
ivGC therapy.
2 Abnormal heart, liver and kidney function.

ivGC, intravenous glucocorticoid

as yes 1 or no 0 . If thyroid function was impaired before treatment and restored after treatment, we scored the restoration of euthyroidism as 1 . If thyroid function was still abnormal or was not impaired at the beginning, a score of 0 was recorded.

\section{Statistical analysis}

Continuous variables were reported as the median \pm SD or median [interquartile intervals, 25 th -75 th percentile], and categorical variables were reported as percentages. Continuous variables were compared using the MannWhitney U-test. Categorical variables were compared using the $X$ [2] test or Mann-Whitney U-test, as appropriate. Simple logistic regression was used to identify potential parameters associated with the effects of ivGC therapy. Multivariable logistic regression was then used to determine statistically significant outcomes. Diagnostic accuracy was evaluated using receiver operating characteristic (ROC) curve analysis. Statistical significance was defined as $p<0.05$. All statistical analyses were performed by two authors and a statistician, using the SPSS 13.0 software (SPSS, Chicago, IL, USA).

\section{Results}

Ninety patients (32 males, 58 females; mean age \pm SD, $44.61 \pm 15.06$ years; age range $15-85$ years) were included in the study. All patients were Asians. Thirty-three (36.7\%) patients presented with a pretreatment CAS $<3 / 7$, but had increased signal intensity of extraocular muscles on contrast-enhanced orbital MRI. Fifty-two (57.8\%) patients who showed a response to ivGC therapy were defined as "responsive," and the other 38 (42.2\%) were defined as "unresponsive". The mean duration of eye symptoms was 8.75 months (range of 4-12 months) in the responsive group, and 24 months (12-75 months) in the unresponsive

Table 3 Overall response

1 Reduction in lid width by at least $3 \mathrm{~mm}$
2 Reduction in any of the class 2 NO SPECS signs by at least two grades
3 Reduction in intraocular pressure by at least $2 \mathrm{mmHg}$
4 Reduction in proptosis by at least $2 \mathrm{~mm}$
5 Improvement in CAS by at least two points
6 Improvement in diplopia (disappearance or lessening of the degree)
7 Improvement in visual acuity by 1 Snellen score

Determination of overall response was based on several studies [5, 7]; NO SPECS was based on the EUGOGO consensus [2]; CAS clinical activity score group, with an OR of 0.984 (95\% confidence interval [CI], $0.971-0.996, p=0.011)$. The mean pretreatment CAS in the two groups were $4(3,4)$ (responsive) and 2 (1-3) (unresponsive), with an OR of 1.653 (95\% CI, 1.214-2.250, $p=$ 0.001). The OR of the restoration of euthyroidism was 3.272 (95\% CI, 1.150-9.315, $p=0.026$ ). Generally, these three factors were identified as parameters that were associated with the effects of ivGC therapy (Table 4). However, gender, age, smoking history, family history of TAO, and a history of impaired thyroid function were not identified as parameters that were associated with the effects of ivGC therapy.

We analyzed the interactions among the three variables. Significant interactions were observed between the pretreatment CAS and both the duration of eye symptoms and restoration of euthyroidism (Table 5). After excluding the pretreatment CAS from our analysis, multivariable logistic regression was performed for the other two variables: the duration of eye symptoms and restoration of euthyroidism.

Both variables were found to be associated with the effects of ivGC therapy (Table 6). The OR of the duration of eye symptoms was 0.984 (95\% CI, 0.972-0.997, $p=0.012$ ), and that of the restoration of euthyroidism was $3.282(95 \%$ CI, $1.062-10.142, p=0.039)$. Therefore, a shorter duration of eye symptoms led to greater efficacy of the therapy. More specifically, the earlier therapy commenced, the more favorable were the effects observed.

The diagnostic accuracy of the duration of eye symptoms was 13 months (95\% CI, 0.641-0.850, $p=0.000$ ), based on ROC curve analysis, with an area under curve (AUC) of 0.746 , specificity of $76.9 \%$ and sensitivity of $65.8 \%$ (Fig. 1a). The diagnostic accuracy of the pretreatment CAS was 2.5 (95\% CI, 0.620-0.836, $p=0.000)$, with an AUC of 0.728 , specificity of $61.5 \%$ and sensitivity of $80.5 \%$ (Fig. 1b). The ROC curve analysis of restoration of euthyroidism showed an AUC of 0.613 (95\% CI, 0.494$0.732, p=0.074$ ) (Fig. 1c). Furthermore, a multivariable prediction model was established with variables of the duration of eye symptoms and restoration of euthyroidism. The ROC curve analysis showed an AUC of 0.784 (95\% CI, 0.685-0.882, $p=0.000$ ) (Fig. 1d), which was better than those single-variable prediction models.

\section{Discussion}

Our study found three potential factors that were associated with the effects of ivGC therapy. A higher CAS indicated a stronger inflammatory reaction and increased disease activity that was likely to be alleviated following ivGC therapy [9]. Interaction analysis revealed significant interactions between the pretreatment CAS and duration of eye symptoms. These two parameters were not independent of each other. These findings are consistent with those from clinical experience: patients were more likely to have inactive TAO with a longer duration 
Table 4 Results of simple logistic regression

\begin{tabular}{|c|c|c|c|c|c|c|}
\hline \multirow[t]{2}{*}{ Factor } & \multirow[t]{2}{*}{ Responsive } & \multirow[t]{2}{*}{ Unresponsive } & \multirow[t]{2}{*}{$p$-Value } & \multirow[t]{2}{*}{ OR } & \multicolumn{2}{|c|}{$95 \% \mathrm{Cl}$ of $\mathrm{OR}$} \\
\hline & & & & & Lower & Upper \\
\hline Gender, males & $21(40.4 \%)$ & $11(28.9 \%)$ & 0.265 & 1.663 & 0.680 & 4.063 \\
\hline Age, years & $46.75 \pm 12.95$ & $41.68 \pm 17.3$ & 0.117 & 1.023 & 0.994 & 1.053 \\
\hline Duration of eye symptoms, months & $8.75[4-12]^{a}$ & 24 [12-75] & 0.011 & 0.984 & 0.971 & 0.996 \\
\hline Smoking history & $28(53.8 \%)$ & $19(50 \%)$ & 0.718 & 1.167 & 0.505 & 2.696 \\
\hline Family history of TAO & $9(17.3 \%)$ & $12(31.6 \%)$ & 0.118 & 0.453 & 0.168 & 1.223 \\
\hline History of impaired thyroid function & $44(84.6 \%)$ & $36(94.7 \%)$ & 0.149 & 0.306 & 0.061 & 1.530 \\
\hline Pretreatment CAS & $4[3-4]$ & $2[1-3]$ & 0.001 & 1.653 & 1.214 & 2.250 \\
\hline Restoration of euthyroidism & $19(36.5 \%)$ & $6(15.8 \%)$ & 0.026 & 3.272 & 1.150 & 9.315 \\
\hline
\end{tabular}

OR Odds ratio, $\mathrm{Cl}$ confidence interval, TAO thyroid-associated ophthalmopathy, CAS clinical activity score, [interquartile intervals, 25th-75th percentile]

of eye symptoms, whereas inactive TAO was characterized by a lower CAS. The existing cut-off point between active TAO and inactive TAO was a CAS of 3 . A CAS $\geq 3 / 7$ was indicative of active TAO [9-11]. The ivGC therapy administered as a form of immunosuppressive therapy proved to be effective against active TAO, and based on the pathogenesis of TAO, was also an acceptable choice [7, 8]. The recent consensus statement of EUGOGO recommended high-dose ivGC as the first-line treatment for patients with a CAS $\geq 3 / 7$ [2]. However, the inclusion criteria of our study were not restricted to a pretreatment CAS $\geq 3 / 7$. Some patients with a CAS $<3 / 7$ exhibited inflammatory signs, such as soft tissue swelling or enlargement of the extraocular muscles on contrast-enhanced MRI. We found glucocorticoids to be generally effective in treating these symptoms. Thus, we decided to include patients with a CAS $<3 / 7$ who exhibited an increased signal intensity of the extraocular muscles on contrast-enhanced MRI in our analysis as well.

Based on the results of ROC analysis, we found that ivGC therapy was more effective when the CAS was $>2.5$. However, these results might have been limited by the relatively small sample size. Furthermore, the criteria established in previous studies $[9,10]$ were not designed for Asian patients. We believe that the cut-off point for the CAS of Asian patients might be $<3$. This is because of the differences in orbital anatomy between Caucasians and Asians; Asian eyelids tend to have more subcutaneous

Table 5 Results of interaction analysis

\begin{tabular}{lllll}
\hline Factor & p-value & OR & \multicolumn{2}{l}{$95.0 \%$ Cl of OR } \\
\cline { 3 - 5 } & & & Lower & Upper \\
\hline $\begin{array}{l}\text { Pretreatment CAS by duration of } \\
\text { eye symptoms }\end{array}$ & 0.002 & 0.983 & 0.972 & 0.994 \\
$\begin{array}{l}\text { Pretreatment CAS by restoration } \\
\text { of euthyroidism }\end{array}$ & 0.020 & 1.453 & 1.059 & 1.992 \\
$\begin{array}{l}\text { Duration of eye symptoms by } \\
\text { restoration of euthyroidism }\end{array}$ & 0.215 & 0.987 & 0.966 & 1.008 \\
\hline
\end{tabular}

OR Odds ratio, $\mathrm{Cl}$ confidence interval, CAS clinical activity score and suborbicularis fat [12]. The thicker eyelid might lead to reduced orbital inflammation. In one Chinese study [7], only $5.13 \%-10.26 \%$ of Asian patients presented with eyelid redness, whereas a Dutch study [10] reported this figure to be less than $50 \%$. Nevertheless, this theory would require further investigation to be confirmed. Randomized controlled trials on larger sample sizes, specifically designed for Chinese patients are recommended for further research.

Interactions were also noted between the pretreatment CAS and restoration of euthyroidism. The reason for this interaction remains unclear, based on our present clinical knowledge. After the parameter of the pretreatment CAS was excluded, and multivariable logistic regression for the other two variables was conducted, we found that both the duration of eye symptoms and restoration of euthyroidism could have affected the effects of ivGC therapy. Patients with a shorter duration of eye symptoms were more likely to be at an active phase of the condition, and were thus more likely to respond to glucocorticoid therapy. We also found that ivGC therapy was more likely to be effective when patients were administered the therapy within 13 months after the first appearance of eye symptoms. Therefore, prompt diagnosis and treatment are recommended.

The restoration of euthyroidism indicated an impairment of thyroid function before treatment and a subsequent restoration before the last follow-up. Thus, a patient with impaired thyroid function became euthyroid following therapy, and was generally more responsive to ivGC therapy. These findings could be attributed to the

Table 6 Results of multivariable logistic regression

\begin{tabular}{lllll}
\hline Factor & p-value & OR & \multicolumn{2}{l}{$95.0 \%$ Cl of OR } \\
\cline { 4 - 5 } & & & Lower & Upper \\
\hline Duration of eye symptoms & 0.012 & 0.984 & 0.972 & 0.997 \\
Restoration of euthyroidism & 0.039 & 3.282 & 1.062 & 10.142 \\
\hline
\end{tabular}

OR Odds ratio, $\mathrm{Cl}$ confidence interval 

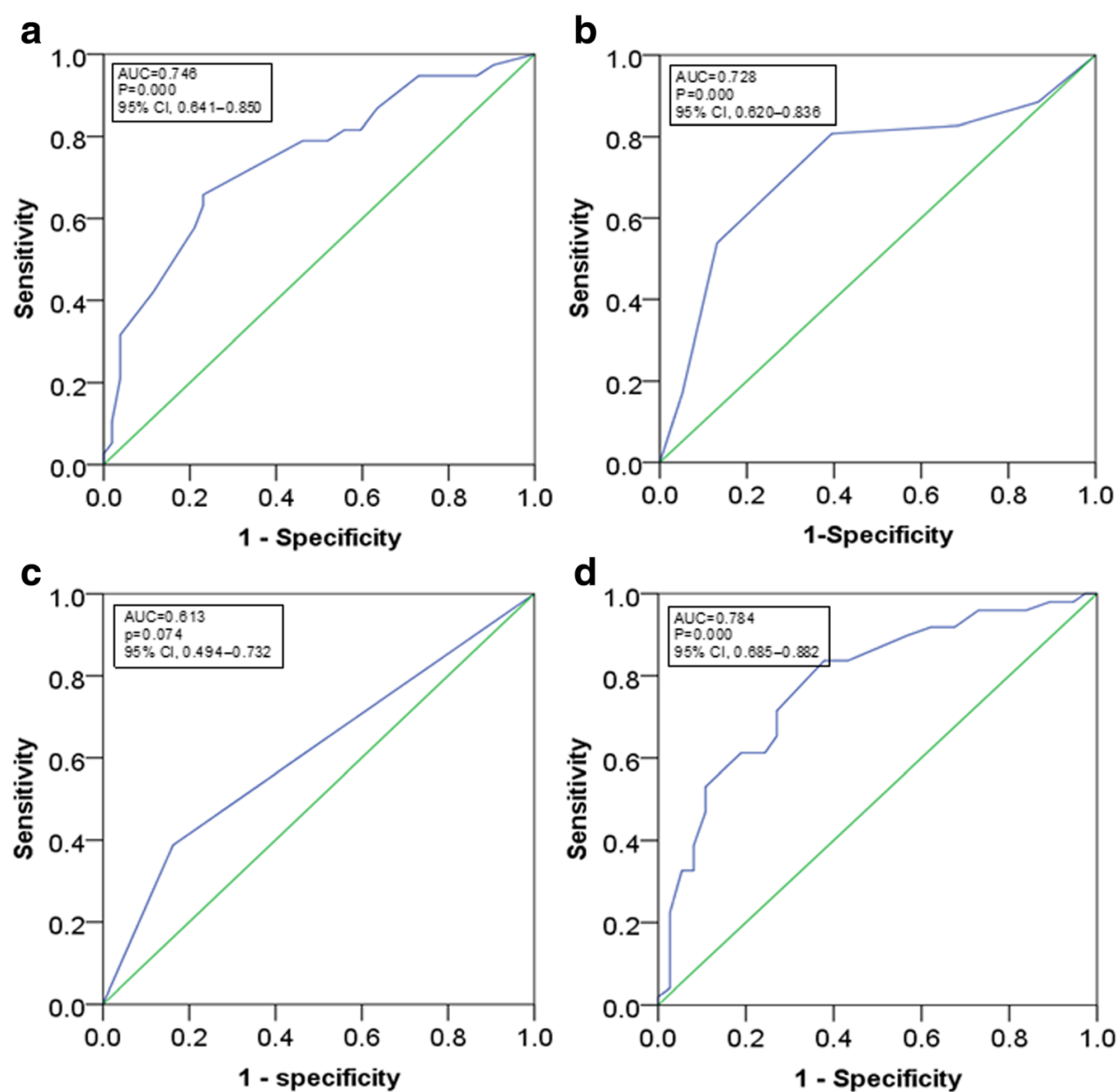

Fig. 1 Receiver operating characteristic (ROC) curve analysis. a duration of eye symptoms; b pretreatment CAS; c restoration of euthyroidism (a binary categorical variable); $\mathbf{d}$ a multivariable prediction model with variables of the duration of eye symptoms and restoration of euthyroidism. $R O C$ receiver operating characteristic, $A \cup C$ area under curve, $C l$ confidence interval, CAS clinical activity score

concurrent administration of anti-thyroid drugs. Methimazole is a common drug indicated for thyroid dysfunction. According to previous studies, it is also an immunosuppressant $[13,14]$. Thus, we speculated that some anti-thyroid drugs could alleviate inflammation of the orbital tissue and improve the overall efficacy of glucocorticoids. Prompt restoration and maintenance of euthyroidism is recommended for TAO patients, both to enhance thyroid function and improve the efficacy of ivGC therapy.

Besides, a multivariable prediction model was established including variables of the duration of eye symptoms and restoration of euthyroidism, which was better in the forecasting aspect than those single-variable prediction models with an AUC of 0.784. We hope to verify this model in larger cohorts. Hope it can be used to predict ivGC efficacy and facilitate the selection of suitable patients.

One study conducted in Italy reported that age and glucocorticoid dose were major risk factors for liver damage, a common side effect of ivGC therapy in TAO [15]. Researchers have also reported that the response to ivGC therapy increases with time [16]. Biomarkers of serum microRNA-224-5p and thyroid-stimulating hormone receptor antibody (TRAb) have been observed to predict glucocorticoid sensitivity [17]. In another study [18], smoking is reportedly associated with a poor response to glucocorticoid therapy. However, this finding is not consistent with the present results. One possible reason might be the different grouping methods. In that study, they divided the patients into three groups: Never-smokers (23 patients), active smokers (31 patients), and passive smokers (38 patients). Patients who had never smoked throughout their lives and had never breathed in the smoke from other people's cigarettes throughout their lives were considered as never smokers. Patients who had never smoked themselves but breathed in the smoke from other people's cigarettes were considered as passive smokers. The others were considered as active smokers. They compared active smokers and never smokers. They found that neversmokers showed a significantly higher response rate than active smokers [18]. In our study, we divided patients in two groups: group 1 included active smokers and group 
2 included never smokers and passive smokers. We compared the two groups and inferred that smoking was not a factor that was significantly associated with the effects of ivGC therapy. Another study also reported that smoking was not a factor that affects the efficacy of ivGC therapy [16]. They divided patients in the same way as ours. Thus, we inferred that passive smokers might have interfered with the analysis. Further research should be conducted to determine the nature of this interference. Another limitation of our study was the follow-up time that was not long enough to determine the long-term effects of glucocorticoid administration.

\section{Conclusion}

In patients with TAO, a shorter duration of eye symptoms ( $\leq 13$ months) and the restoration of euthyroidism indicated greater efficacy of ivGC therapy. The pretreatment CAS was not an independent factor, but was an important parameter for the prediction of efficacy and selection of suitable patients. A multivariable prediction model had been established. We hope it can be applied to clinical therapy in the future. Based on the present results and other recent studies, active smoking history could also be a potential factor that can affect the efficacy of ivGC therapy.

\section{Abbreviations}

AUC: area under curve; CAS: clinical activity score; Cl: confidence interval; EUGOGO: European Group on Graves' Orbitopathy; ivGC: intravenous glucocorticoid; MRD: margin reflex distance; MRI: magnetic resonance imaging; OR: odds ratio; ROC: receiver operating characteristic; TAO: thyroid-associated ophthalmopathy; TRAb: thyroid-stimulating hormone receptor antibody

\section{Acknowledgments}

Thanks Dr. Tienan Feng of Clinical Research Center, Shanghai Jiao Tong University Medicine School for the assistance of medicine statistics. This work was supported by The Science and Technology Commission of Shanghai (17DZ2260100).

\section{Funding}

This study was supported in part by the National High Technology Research and Development Program (863 Program) (2015AA020311), NSFC grants 31600971, 81300799, 81320108010, 81170876, 31271029, 81570883; Shanghai Municipality Commission for Science and Technology (14JC1493103, 12419A9300); Shanghai Municipal Education Commission-Gaofeng Clinical Medicine Grant Support (20152228); Shanghai Municipal Hospital Emerging Frontier Technology Joint Research Project (SHDC12012107); and Shanghai JiaoTong University Medical \& Engineering Cross Fund (YG2014MS03).

\section{Availability of data and materials}

The datasets used and analyzed during the current study are available from the corresponding author on reasonable request.

\section{Authors' contributions}

YW, HZ, and XF designed the study. YW and $\mathrm{HZ}$ analyzed and interpreted the patients' data regarding thyroid-associated ophthalmopathy and were major contributors in writing the manuscript. SZ, YZ, XL, HG, SZ, YH, and SF performed the ophthalmic assessments and made substantial contributions to acquisition of data. JS, HZ, and XF reviewed and edited the manuscript. All authors read and approved the final manuscript.

\section{Ethics approval and consent to participate}

This retrospective study was approved by the Ethics Committee of Shanghai Ninth People's Hospital. The written informed consents were obtained from all participants. This study was in compliance with the tenets of the Declaration of Helsinki for clinical research.
Consent for publication

Not applicable

\section{Competing interests}

The authors declare that they have no competing interests.

\section{Publisher's Note}

Springer Nature remains neutral with regard to jurisdictional claims in published maps and institutional affiliations.

\section{Author details}

'Department of Ophthalmology, Ninth People's Hospital, Shanghai JiaoTong University School of Medicine, No. 639 ZhiZaoJu Road, Shanghai 200011, China. ${ }^{2}$ Shanghai Key Laboratory of Orbital Diseases and Ocular Oncology, Shanghai, China. ${ }^{3}$ Shanghai Jiao Tong University School of Medicine, Shanghai, China

Received: 23 August 2017 Accepted: 13 February 2018

Published online: 20 February 2018

\section{References}

1. Bartley GB, Fatourechi V, Kadrmas EF, Jacobsen SJ, Ilstrup DM, Garrity JA, et al. Clinical features of graves' ophthalmopathy in an incidence cohort. Am J Ophthalmol. 1996;121:284-90.

2. Bartalena L, Baldeschi L, Boboridis K, Eckstein A, Kahaly GJ, Marcocci C, et al. The 2016 European thyroid association/European group on Graves' Orbitopathy guidelines for the Management of Graves' Orbitopathy. Eur Thyroid J. 2016:5:9-26.

3. Rao R, Macintosh PW, Yoon MK, Lefebvre DR. Current trends in the management of thyroid eye disease. Curr Opin Ophthalmol. 2015;26:484-90.

4. Marcocci C, Bartalena L, Tanda ML, Manetti L, Dell'Unto E, Rocchi R, et al. Comparison of the effectiveness and tolerability of intravenous or oral glucocorticoids associated with orbital radiotherapy in the management of severe graves' ophthalmopathy: results of a prospective, single-blind, randomized study. J Clin Endocrinol Metab. 2001:86:3562-7.

5. Kahaly GJ, Pitz S, Hommel G, Dittmar M. Randomized, single blind trial of intravenous versus oral steroid monotherapy in graves' orbitopathy. J Clin Endocrinol Metab. 2005;90:5234-40.

6. Marcocci C, Bartalena L, Panicucci M, Marconcini C, Cartei F, Cavallacci G, et al. Orbital cobalt irradiation combined with retrobulbar or systemic corticosteroids for graves' ophthalmopathy: a comparative study. Clin Endocrinol. 1987;27:33-42.

7. Zhu W, Ye L, Shen LY, Jiao Q, Huang F, Han R, et al. A prospective, randomized trial of ivGC therapy with different protocols for patients with graves' ophthalmopathy. J Clin Endocrinol Metab. 2014;99:1999-2007.

8. Zang S, Ponto KA, Kahaly GJ. Clinical review: ivGC for graves' orbitopathy: efficacy and morbidity. J Clin Endocrinol Metab. 2011;96:320-32.

9. Mourits MP, Koornneef L, Wiersinga WM, Prummel MF, Berghout A, van der Gaag R. Clinical criteria for the assessment of disease activity in graves' ophthalmopathy: a novel approach. Br J Ophthalmol. 1989;73:639-44.

10. Mourits MP, Prummel MF, Wiersinga WM, Koornneef L. Clinical activity score as a guide in the management of patients with graves' ophthalmopathy. Clin Endocrinol. 1997:47:9-14.

11. Bartalena L, Baldeschi L, Dickinson A, Eckstein A, Kendall-Taylor P, Marcocci $C$, et al. Consensus statement of the European group on graves' orbitopathy (EUGOGO) on management of GO. Eur J Endocrinol. 2008;158:273-85.

12. Jeong S, Lemke BN, Dortzbach RK, Park YG, Kang HK. The Asian upper eyelid: an anatomical study with comparison to the Caucasian eyelid. Arch Ophthalmol. 1999:117:907-12.

13. Michie W, Bech JS, Mahaffy RG, Honein EF, Fowler GB. Quantitative, radiological and histological studies of the thymus in thyroid disease. Lancet. 1979;1:691-5.

14. Balazs C, Kiss E, Leövey A, Farid NR. The immunosuppressive effect of methimazole on cell-mediated immunity is mediated by its capacity to inhibit peroxidase and to scavenge free oxygen radicals. Clin Endocrinol. 1986;25:7-16.

15. Sisti E, Coco B, Menconi F, Leo M, Rocchi R, Latrofa F, et al. Age and dose are major risk factors for liver damage associated with intravenous glucocorticoid pulse therapy for Graves' Orbitopathy. Thyroid. 2015;25:846-50.

16. Leo M, Mautone T, Ionni I, Profilo MA, Sabini E, Menconi F, et al. Variables affecting the long term outcome of Graves' orbitopathy following high 
dose intravenous glucocorticoid pulse therapy in patients not treated with orbital radiotherapy. Endocr Pract. 2016;22:1177-86.

17. Shen L, Huang F, Ye L, Zhu W, Zhang X, Wang S, et al. Circulating microRNA predicts insensitivity to glucocorticoid therapy in Graves' ophthalmopathy. Endocrine. 2015;49:445-56.

18. Xing L, Ye L, Zhu W, Shen L, Huang F, Jiao Q, et al. Smoking was associated with poor response to intravenous steroids therapy in Graves' ophthalmopathy. Br J Ophthalmol. 2015;99:1686-91.

Submit your next manuscript to BioMed Central and we will help you at every step:

- We accept pre-submission inquiries

- Our selector tool helps you to find the most relevant journal

- We provide round the clock customer support

- Convenient online submission

- Thorough peer review

- Inclusion in PubMed and all major indexing services

- Maximum visibility for your research

Submit your manuscript at www.biomedcentral.com/submit 